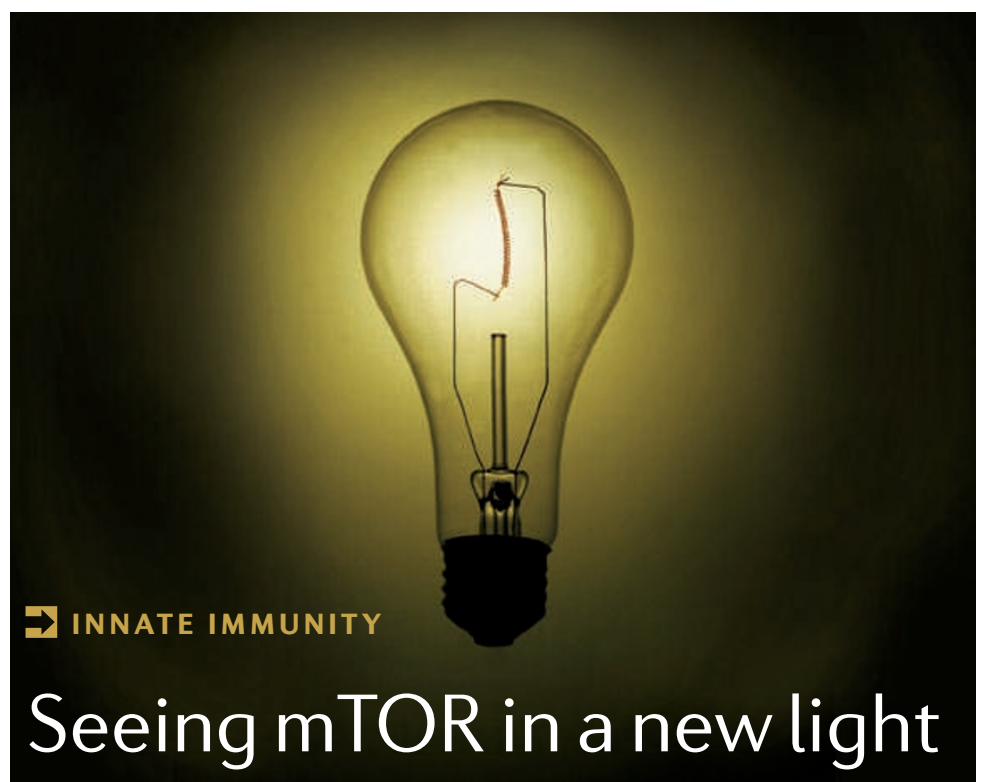

A recent study published in Immunity shows that the drug rapamycin has a surprisingly pro-inflammatory effect on phagocytic cells, increasing their capacity to produce cytokines, such as interleukin-12 (IL-12), and to prime $\mathrm{T}$ helper $1\left(\mathrm{~T}_{\mathrm{H}} 1\right)$ - and $\mathrm{T}_{\mathrm{H}} 17$-cell responses.

Rapamycin is used to prevent graft rejection in transplant recipients based on its ability to suppress T-cell proliferation. The drug blocks the activity of a protein known as mammalian target of rapamycin (mTOR), which promotes proliferation through the activation of proteins such as S6 kinase. Although the mTOR pathway has been characterized in T cells, it has not been well described in other immune cells. In this study, Weichhart et al. investigated how mTOR activity in phagocytes regulates the innate inflammatory response.

The mTOR pathway is activated in phagocytes in response to bacterial infection or following exposure to lipopolysaccharide (LPS). Unexpectedly, rapamycinmediated inhibition of mTOR caused an increase in the production of pro-inflammatory cytokines, such as IL-12, IL-23 and IL-6, and a decrease in the production of the anti-inflammatory cytokine IL-10 following LPS stimulation of primary human phagocytes. The opposite effect was observed in cells that were deficient for the tuberous sclerosis complex (TSC; an endogenous negative regulator of mTOR activity), in which mTOR is hyperactive: $T s c 2^{-/-}$ cells produced less IL-12 and more IL-10 following LPS stimulation compared with wild-type cells. So, the TSC-mTOR pathway negatively regulates the innate inflammatory response, and rapamycin-mediated inhibition of mTOR prevents a downregulation of the production of pro-inflammatory cytokines in LPS-stimulated phagocytes.

Although mTOR is thought to influence cytokine expression in T cells mainly by affecting mRNA stability, molecular analyses showed that mTOR regulates cytokine production in phagocytes at the transcriptional level through at least two independent mechanisms. mTOR was shown to limit the production of pro-inflammatory cytokines by

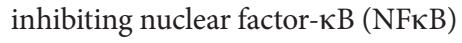
activity, whereas it promoted IL-10 production by activating signal transducer and activator of transcription 3 (STAT3), which is necessary for the expression of this cytokine.

The pro-inflammatory effect of rapamycin on innate immune cells was also observed when the capacity of monocytes to stimulate T cells was tested. Although rapamycin could act directly on T cells to suppress their proliferation and effector function, it increased the ability of LPSstimulated monocytes to prime $\mathrm{T}_{\mathrm{H}} 1$ and $\mathrm{T}_{\mathrm{H}} 17$ cells in vitro.

The physiological relevance of mTOR inhibition in phagocytes was also tested in vivo using a model of Listeria monocytogenes infection of $\mathrm{BALB} / \mathrm{c}$ mice, in which this pathogen is fatal. Pre-treatment with rapamycin for 3 days markedly increased the survival of mice that had been infected with L. monocytogenes, an effect that depended on phagocytes and correlated with an increase in the levels of IL-12, IL-6 and interferon- $\gamma$ in the serum.

These data show that the TSC-mTOR pathway is a crucial regulator of the innate inflammatory response, which might help to explain the inflammatory side effects that can occur in transplant recipients treated with rapamycin. In addition, this study also supports the finding that rapamycin has beneficial effects in patients with cancer, in whom boosting the innate inflammatory response might promote antitumour immunity.

\section{Sarah Allan}

ORIGINAL RESEARCH PAPER Weichhart, T. et al. The TSC-mTOR signaling pathway regulates the innate inflammatory response. Immunity $\mathbf{2 9}$, 565-577 (2008) 